\title{
Funcionalidade da relação entre habilidades sociais e dificuldades de aprendizagem
}

\author{
Renata Cristina Moreno Molina \\ Zilda Aparecida Pereira Del Prette ${ }^{1}$
}

\begin{abstract}
Resumo
Estudos no campo das habilidades sociais têm demonstrado a existência de relações entre desempenho interpessoal e acadêmico, embora ainda haja contradições quanto à natureza e funcionalidade dessa relação. O presente estudo pretendeu examinar a relação empírica entre esses dois aspectos por meio de: a) promoção das habilidades sociais com avaliação dos efeitos sobre o repertório acadêmico e b) promoção do repertório acadêmico com avaliação dos efeitos sobre o repertório de habilidades sociais. Utilizou-se um delineamento quase experimental de grupo (um grupo controle e dois experimentais), avaliando-se o repertório acadêmico e social dos alunos antes e após a intervenção. Participaram 16 estudantes com dificuldades na aprendizagem de leitura e escrita, cujas idades variavam entre sete e treze anos. O Treinamento em Habilidades Sociais consistiu em atividades lúdico-pedagógicas com objetivos organizados em ordem crescente de complexidade. A intervenção acadêmica caracterizou-se pelo ensino de 51 palavras de duas e três sílabas simples. Do repertório acadêmico registrou-se leitura, nomeação e ditado. As habilidades sociais foram avaliadas pelo próprio participante e por seus professores e colegas. Os resultados mostraram que, enquanto o grupo que passou por intervenção acadêmica apresentou ganhos em leitura e escrita, o grupo que passou pela intervenção em habilidades sociais apresentou ganhos no repertório social e no acadêmico. Os resultados favoreceram a idéia da existência da relação funcional entre habilidades sociais e acadêmicas.

Palavras-chave: Dificuldades de aprendizagem; Leitura-escrita; Habilidades sociais.
\end{abstract}

\section{Functionality of relations between social skills and learning disabilities}

\begin{abstract}
Studies in social skills area have established relations between social and academic performance, although the data regarding the nature and the functionality of this relationship remain somewhat controversial. The present study aimed to evaluate the empirical relationship between these two aspects through two pathways: a) promoting social skills and evaluating their effects on academic results; b) promoting academic results and evaluating their effects on social skills. An experimental group design was adopted (one control and two experimental groups) with students' academic and social skills evaluated before and after the intervention. Sixteen 7 to 13 years-old students, with reading and writing learning disabilities participated. The Social Skills Training Program consisted of pedagogical play activities organized from a lower to a higher level of complexity goals. The academic intervention consisted of teaching 51 words of two and three simple syllables. The students' academic results were assessed by the evaluation of reading, naming and dictation skills. The social skills results were assessed by self-evaluation and peer and teacher evaluation. The results showed that the academic intervention group produced reading and writing skills improvement. On the other hand, the social-skills intervention group improved both social and academic skills. The results reinforce the idea of a functional relationship between social and academic abilities.
\end{abstract}

Keywords: Learning disabilities; Reading-writing; Social skills.

\section{Introdução}

O fracasso escolar é um fenômeno recorrente na história educacional brasileira, constituindo-se um crônico e permanente desafio para psicólogos, pedagogos e profissionais de áreas afins. Apontado como o responsável pela exclusão, da escola, de importantes segmentos da população, o fracasso escolar foi explicado com base em diferentes aspectos nas últimas décadas. Até a década de 70, por exemplo, a ideologia do discurso liberal e neoliberal situava a ênfase do fracasso escolar em fatores extra-escolares, como características do aluno e de sua família, sendo a desigualdade acadêmica atribuída a fatores pessoais. A partir dos anos 70, a explicação para o fracasso escolar deslocou-se da esfera biológica para a cultural, centrando-se na defasagem sociocultural que as

\footnotetext{
${ }^{1}$ Endereço para correspondência:

UFSCar - Departamento de Psicologia

Via Washington Luiz - km 235 - Bairro Monjolinho - 13565-905 - São Carlos-SP

E-mail: zdprette@power.ufscar.br
} 
crianças, particularmente as de baixa renda, possuíam ao ingressar na escola (Almeida, Rabelo, Cabral, Moura, Barreto, \& Barbosa, 1995).

Em uma revisão atualizada sobre o tema, Sánchez (2004) cita autores que estabelecem uma diferenciação entre os alunos que sofrem fracasso escolar, caracterizado como um baixo rendimento, devido a causas extrínsecas (ensino inadequado, baixa motivação e fatores econômicos), dos que sofrem dificuldades de aprendizagem originadas por fatores intrínsecos (discrepâncias significativas no desenvolvimento dos processos psicológicos como a percepção, a atenção e a memória). Tendo em vista os fatores que podem estar associados às dificuldades de aprendizagem, a concepção que norteia o presente trabalho as considera como decorrentes de uma constelação de fatores de ordem pessoal, familiar, emocional, pedagógica e social que só adquire sentido quando referida à história de relações e interações do sujeito com seu meio, sobretudo, o escolar (Almeida et al., 1995).

Independentemente de qual seja a causa do problema que gera a limitação da aprendizagem, deve-se considerar que tais alunos apresentam necessidades educativas especiais e, portanto, requerem atendimento especializado, oferecido pela Educação Especial, para o desenvolvimento de todos os aspectos de sua vida (Sánchez, 2004).

O Ministério da Educação, por meio do documento Política Nacional da Educação Especial (Brasil, 1994), define como alunado da Educação Especial aquele que, por apresentar necessidades próprias e diferentes dos demais alunos no domínio das aprendizagens curriculares correspondentes à sua idade, requer recursos pedagógicos e metodologias educacionais específicas. São incluídas nesta categoria, pessoas com necessidades educativas especiais, ou seja, os indivíduos com deficiência (mental, visual, auditiva, física e múltipla), com condutas típicas (problemas de conduta) e com altas habilidades (superdotados). As crianças com dificuldades de aprendizagem não são contempladas na visão oficial desta área e, para que possam usufruir o ensino especializado de que necessitam, recebem o rótulo de "deficientes mentais" e são encaminhadas do ensino regular para as classes especiais, sendo discriminadas e marginalizadas (Almeida, 1984).

A Secretaria de Educação Especial (Brasil, 1994) aponta a necessidade de se repensar esses encaminhamentos propondo aos alunos com dificuldades de aprendizagem um atendimento especializado, que deve ocorrer na rede regular de ensino, com apoio pedagógico específico. Souza e Machado (1997) apontam a necessidade de a Educação Especial assumir um novo conceito, configurando-se como o conjunto de apoio disponível não somente às necessidades especiais associadas às deficiências, aos problemas de conduta ou superdotação, mas também aos alunos com dificuldades de aprendizagem, que também necessitam de recursos específicos para o ideal desenvolvimento de suas potencialidades. Essa proposta, coerente com os princípios da Educação Inclusiva, tem como desafio estender $\mathrm{o}$ atendimento a toda a população, com ações que impliquem na inclusão de todos os alunos, quaisquer que sejam suas limitações e possibilidades individuais e sociais (Nunes, Ferreira, Glat, \& Mendes, 1998). A inserção de alunos com necessidades educativas especiais na sala regular da escola comum traria benefícios a todos, indiscriminadamente, no sentido de proporcionar uma formação mais humana, coletiva, democrática e sem preconceitos.

No entanto, mesmo sob esta nova condição, ainda são comuns as queixas de dificuldades de aprendizagem e o encaminhamento desses alunos para o Ensino Especial, indicando uma possível falência do sistema regular. De acordo com Hildebrand (2000), essa falência ocorre porque as mudanças na estrutura geral do ensino não têm sido acompanhadas por melhorias significativas no processo de ensino-aprendizagem. Essa autoria entende que tais melhorias exigiriam a alteração das condições de assimilação dos conteúdos acadêmicos e que uma das maneiras de se conseguir isso seria por meio do aperfeiçoamento dos métodos de ensino e das condições de desenvolvimento interpessoal dos alunos, o que requer o estabelecimento de interações sociais produtivas e satisfatórias entre os alunos e seu meio, sobretudo o escolar.

As interações sociais são entendidas como educativas na medida em que representam condições para a aquisição de conceitos, habilidades e estratégias cognitivas que afetam o desenvolvimento social e a aprendizagem (Coll \& Sole, 1995; Del Prette \& Del Prette, 1997a; Pontecorvo, s.d.). As interações sociais satisfatórias da criança, com colegas e professores, requerem um repertório adequado de habilidades sociais, ou seja, de diferentes classes de comportamentos sociais para lidar de maneira adequada com as demandas das situações interpessoais (Del Prette \& Del Prette, 2001; 2003; 2005a). As dificuldades em habilidades sociais, definidas como déficits de aquisição ou de desempenho adequado às demandas da situação e da cultura, interferem, portanto, na qualidade dessas relações (Del Prette \& Del Prette, 2005a; Gresham, 1992; 1995; Malecki \& Elliott, 2002; McClelland, Morrison, \& Holmes, 2000).

Assim, considerando que o conceito de habilidades sociais parece ser adequado e central para analisar as interações sociais e considerando que a aprendizagem é um processo de construção social do

Psico-USF, v. 11, n. 1, p. 53-63, jan./jun. 2006 
conhecimento que ocorre na interação do sujeito com seu meio, é razoável supor a existência de relações entre dificuldades de aprendizagem e déficits em habilidades sociais. Pesquisas nesta área (Del Prette \& Del Prette, 2003; Gresham, 2002; O’Shaughnessy, Lane, Gresham, \& Beebe-Frankenberger, 2002; Romero, 1995) têm mostrado que as crianças com distúrbios ou dificuldades de aprendizagem apresentam características interpessoais que incluem, entre outros aspectos, tendência a serem mais agressivas, a apresentarem interações mais negativas com companheiros, a terem mais problemas de personalidade, menos comportamentos orientados para tarefa e a apresentarem um repertório menos elaborado de comportamentos interpessoais apropriados $\mathrm{e}$ desejáveis socialmente.

Com relação aos pais, constata-se uma avaliação menos positiva destes sobre o repertório social dos filhos com dificuldades de aprendizagem, em comparação com crianças sem essas dificuldades (Feitosa, 2003; Marturano, Linhares \& Parreira, 1993; Mello, 2004; Pinheiro, Haase, Del Prette, Amarante \& Del Prette, s. d.; Romero, 1995), em aspectos como autocontrole, ansiedade, impulsividade e habilidades verbais, modos inadequados de enfrentamento (irritação, resistência, agressão/ataque, ansiedade, oposição e afastamento), tendência a comportamentos pouco adaptativos (agitação, apego, desorganização e esquiva social) e alterações funcionais (do sono, da fala etc.).

Na avaliação dos professores (Fad, 1989; Maluf \& Bardelli 1991; Marturano, \& Loureiro, 2003), os alunos com dificuldades de aprendizagem são referidos como inquietos, briguentos, inibidos e sem iniciativa, com déficits nas habilidades de desenvolver e manter amizades, encerrar conversação, compartilhar brincadeiras e interagir com colegas. Diferentes estudos enfocando a avaliação pelos colegas também demonstraram que os alunos com distúrbios de aprendizagem são menos aceitos que seus colegas sem essas dificuldades (Stone \& La Greca, 1990; Vaughn \& Hogan, 1990; Vaughn, McIntosh, \& SpencerRowe, 1991).

Cabe, no entanto, lembrar que, ao lado das evidências de que os déficits em habilidades sociais podem contribuir para um pobre ajustamento escolar e baixo rendimento acadêmico (Fad, 1989; Romero, 1995; Stone \& La Greca, 1990; Vaughn \& Hogan, 1990), há também estudos mostrando que crianças com distúrbios de aprendizagem podem não apresentar deficiências nas habilidades sociais (Elliott, Pring, \& Bunning, 2002; Gresham, 1992, 2002; Swanson \& Malone, 1992).

Essa controvérsia coloca a questão da natureza da relação entre habilidades sociais e dificuldades de aprendizagem. Gresham (1992) sugere três hipóteses que podem estar norteando os estudos sobre essa relação: a) a

Psico-USF, v. 11, n. 1, p. 53-63, jan./jun. 2006 hipótese causal, na qual os déficits em habilidades sociais e a incapacidade de aprendizado seriam atribuídos à disfunção do sistema nervoso central (o autor constata escassez de evidência empírica apoiando essa hipótese); b) a hipótese da concomitância entre deficiência em habilidades sociais e dificuldades de aprendizado (o autor assinala que déficits sociais ocorrem com uma parcela de alunos de bom desempenho acadêmico e, inversamente, há uma parcela de alunos com dificuldades de aprendizagem com indicadores de desempenho social satisfatório); e c) a hipótese correlacional (o autor salienta que os estudos encontram correlação positiva, mas apenas moderada, entre habilidades sociais e desempenho acadêmico, ou seja, uma tendência de parte dos alunos com maiores escores em habilidades sociais apresentarem melhor rendimento acadêmico). $\mathrm{O}$ autor alerta, no entanto, que o tratamento mais confiável para entender a relação entre déficits em habilidades sociais e distúrbios de aprendizagem consistiria na análise da funcionalidade dessa relação.

A relação funcional entre duas ou mais variáveis ocorre quando estas possuem uma variação de participação baseada na noção de probabilidade condicional (Haynes \& O’Brien, 1990): isola-se um único evento e demonstra-se se este é necessário e suficiente para produzir mudanças no segundo evento. Portanto, para estabelecer uma relação funcional entre habilidades sociais e dificuldades de aprendizagem, seria necessário promover ganhos acadêmicos para verificar os efeitos no repertório social e promover ganhos sociais para verificar os efeitos no acadêmico.

É nessa direção que se encaminhou o presente trabalho, buscando avaliar, em crianças com dificuldades de aprendizagem, a relação entre repertório social e acadêmico por meio de um delineamento quaseexperimental que incluiu: a) a promoção de habilidades sociais em um grupo, visando averiguar seus eventuais efeitos sobre o repertório acadêmico e b) a promoção de repertório acadêmico em outro, visando averiguar seus efeitos sobre o repertório de habilidades sociais.

\section{Método}

\section{Participantes $^{2}$}

A amostra consistiu de 16 estudantes com dificuldades na aprendizagem de leitura e escrita (seis meninas e dez meninos), de duas escolas de periferia do Ensino Fundamental de São Carlos. Foram selecionados para participar do trabalho somente alunos

\footnotetext{
${ }^{2}$ A pesquisa foi aprovada pelo Comitê de Ética em Pesquisa (Protocolo UFSCar 019/2002).
} 
que apresentaram ausência completa de leitura em uma tarefa com quinze palavras. Após a seleção e autorização dos pais, esses alunos foram distribuídos em três grupos: Grupo de Habilidades Sociais (GHS), que passou pelo Treinamento em Habilidades Sociais, Grupo de Ensino de Leitura (GEL), que passou pelo Programa de Ensino de Leitura e Escrita, e Grupo Controle (GC), sem intervenção. O GHS era composto por quatro alunos da Escola 1 (média da idade=10, 25; desvio padrão=2,5), o GEL por quatro alunos da Escola 2 (média da idade $=10$; desvio padrão=1,41) e o GC por oito participantes, sendo quatro de cada escola (média da idade=9,13; desvio padrão=1,64). Todos os grupos foram avaliados antes e após a intervenção quanto ao repertório acadêmico e ao de habilidades sociais. Os participantes do GC estavam nas mesmas salas de aula dos participantes experimentais.

Local

O Programa de Ensino de Leitura e Escrita foi conduzido na Unidade de Iniciação à Leitura, localizada na Biblioteca da UFSCar. O Treinamento em Habilidades Sociais foi conduzido em uma sala da Escola 2, ampla, arejada e praticamente isenta de ruídos externos. As avaliações do repertório acadêmico e de habilidades sociais do GEL e do GC (Escola 1) foram realizadas na Unidade de Iniciação à Leitura e em salas disponíveis da Escola 1. As avaliações do repertório acadêmico e de habilidades sociais do GHS e GC (Escola 2) foram realizadas em salas disponíveis da Escola 2.

\section{Instrumentos e materiais}

Inventário Multimídia de Habilidades Sociais para Crianças IMHSC-Del-Prette (Del Prette \& Del Prette, 2005b). Esse instrumento, aprovado pelo Conselho Federal de Psicologia, consiste em um sistema de avaliação que inclui a auto-avaliação (com os recursos multimídia em Cd-Rom) e a avaliação pelo professor (em versão impressa). A versão multimídia (utilizada no presente estudo) é composta por arquivos de vídeo, contendo 21 seqüências filmadas que retratam situações do cotidiano escolar de crianças do ensino fundamental ( $1^{a}$ à $4^{a}$ séries $)$ em suas interações com pares e com adultos. Cada situação cria, portanto, um contexto onde determinada habilidade social é requerida e, para cada uma delas, são apresentadas à criança três possibilidades de reações: uma habilidosa, uma passiva e uma agressiva. O CdRom é acompanhado de um "Caderno de Aplicação", que orienta a explicação de cada situação às crianças, $\mathrm{e}$ de fichas compostas por 21 números, com três quadrados cada, nos quais o aluno registra a freqüência de emissão das três reações em termos de "sempre", "às vezes" ou "nunca". O Manual apresenta dados psicométricos obtidos com uma amostra ampla de crianças de escolas públicas (alfa de Cronbach $=0,6413$ para freqüência, ${ }^{3}$ correlação positiva e significativa entre teste e reteste e estrutura fatorial com três fatores para freqüência com coeficientes alfa variando de 0,7241 a 0,3058 e indicadores de validade convergente e discriminante).

Ficha de avaliação sociométrica. Esta ficha foi elaborada especialmente para este estudo e consta de uma lista com os nomes das crianças da sala (incluindo as participantes da pesquisa), uma para cada criança, com colunas para indicação dos colegas de quem cada uma mais gosta ou não gosta. Não foi realizada uma análise da consistência interna dos dados produzidos com esta ficha. Instrumentos desse tipo têm sido pouco estudados em termos de propriedades psicométricas, embora se disponha de um procedimento específico (Coie, Dodge \& Copotelli, 1982) para classificar, com base nesses dados, o status sociométrico das crianças em relação aos colegas do grupo/classe.

Protocolo de avaliação da competência social do professor (PACS-P, Del Prette \& Del Prette, 2003). Composto por nove itens, cada um deles com par de adjetivos dicotômicos e um ponto intermediário, que o professor avalia e seleciona a posição que melhor caracteriza o aluno sob avaliação: 1) desinibido - retraído - mais ou menos; 2) querido - rejeitado pelos colegas - mais ou menos; 3) briguento - calmo - mais ou menos; 4) alegre - triste - mais ou menos; 5) faz - não faz perguntas - mais ou menos; 6) ajuda - não ajuda os colegas - mais ou menos; 7) bem - mal educado - mais on menos; 8) disciplinado- indisciplinado - mais ou menos; 9) querido - rejeitado pelos professores - mais ou menos. Em outro estudo (Del Prette, Del Prette, \& Gresham, s. d.) com amostra semelhante à da presente pesquisa, encontrouse que a confiabilidade (alfa de Cronbach) dessa escala foi de 0,778 .

Ficha de avaliação de leitura e escrita (Fonseca, 1997). Instrumento que testa as habilidades que caracterizam os repertórios de leitura e escrita. Neste trabalho foram testadas: a habilidade de seleção/AC (selecionar palavra ouvida), a de nomeação/CD (nomear palavra impressa) e as de ditado/AE (selecionar letra mediante a palavra ditada) e $\mathrm{AF}$

\footnotetext{
${ }^{3}$ Em estudo mais recente com amostragem semelhante, mais ampliada, e uma aplicação mais rigorosamente de acordo com as prescrições do Manual, os dados de consistência interna para freqüência foram de 0,75 a 0,82 para as diferentes reações (Magalhães, Del Prette, \& Del Prette, 2005).
}

Psico-USF, v. 11, n. 1, p. 53-63, jan./jun. 2006 
(escrever mediante a palavra ditada). Cada uma destas tarefas abrange 15 tentativas. Não se dispõe, ainda, de estudos sobre as qualidades psicométricas deste instrumento, nem padrões normativos para os resultados por ele produzidos, mas estudos experimentais (de Rose, de Souza, Rossito, \& de Rose, 1992; Fonseca, 1997; Garotti, de Souza, de Rose, Molina, \& Gil, 2000) têm mostrado sensibilidade aos efeitos de intervenções pedagógicas voltadas para a alfabetização das crianças (Pasquali, 1996). Além disso, trata-se de um instrumento de avaliação de desempenho em que o critério de escore máximo é o desejável e colocado como alvo dessas intervenções.

Para avaliar as habilidades sociais foram ainda utilizados os seguintes materiais: a) microcomputadores e caixas de som; b) materiais pedagógicos como brinquedos, jogos, $\mathrm{CD}$, aparelho de $\mathrm{CD}$, cartolina, papel sulfite, revistas, gibis, lápis de cor, giz de cera, tinta, massinha de modelar, tesoura, cola etc., c) filmadora, tripé e fitas VHS; d) roteiro de planejamento de cada sessão. Para avaliar e promover o repertório acadêmico foram utilizados os materiais: microcomputadores PC, equipados com tela sensível ao toque (Mitsubishi, "diamond 17"), caixas de som, fones de ouvido, papel e lápis preto.

\section{Procedimento de promoção das habilidades sociais}

O Treinamento em Habilidades Sociais ${ }^{4}$ abrangeu 40 sessões de uma hora e meia cada em sete meses de intervenção (60 horas), 30 fora da sala de aula e $10 \mathrm{em}$ sala de aula com todos os alunos. O número de sessões realizadas pelos participantes foi o mesmo. Os objetivos do treinamento foram estabelecidos após a análise dos resultados obtidos pelos alunos do GHS na pré-intervenção (IMHSC-Del Prette e PACS-P).

Para a condução das atividades fora da classe, uma sala da escola era previamente preparada com cadeiras e mesas, com o material a ser utilizado, filmadora e tripé. As dez primeiras sessões foram conduzidas duas vezes por semana em horário alternativo ao período escolar freqüentado pelos alunos. Para a realização das 20 últimas sessões os alunos do GHS eram retirados da classe. Além desses alunos, nestas 20 últimas sessões foram incluídos outros colegas, sem dificuldades de aprendizagem, que os próprios alunos do GHS escolhiam. A inclusão desses novos alunos nas sessões foi uma tentativa de promover a generalização das aquisições do GHS.

\footnotetext{
${ }^{4}$ Uma descrição mais detalhada do procedimento pode ser encontrada em Molina (2003).
}

Psico-USF, v. 11, n. 1, p. 53-63, jan./jun. 2006
Foram utilizados procedimentos lúdicopedagógicos associados a técnicas de modelação, instrução, solução de problemas, feedback, role-playing, reforçamento etc. (Del Prette \& Del Prette, 2001, 2005a; Gresham, 1995, 2002). Em cada sessão era utilizada uma ficha na qual se registravam as áreas deficitárias do repertório de cada participante para orientar alterações nos procedimentos de intervenção, porém o esquema geral do programa foi elaborado com base nas avaliações iniciais. A estrutura do programa de habilidades sociais foi previamente planejada tendo em vista as orientações de Del Prette \& Del Prette (1999; 2001; 2005a) quanto à organização das habilidades consideradas deficitárias em ordem crescente de complexidade em três partes: inicial - verificação de tarefas de casa, fornecimento de avisos e atividades de "aquecimento" que já podiam incluir algumas habilidades (observação, atenção etc.); central aplicação de procedimentos relacionados aos objetivos do programa, por exemplo, treino em elogiar, perguntar, discordar etc.; final - avaliação da sessão, relaxamento e atribuição de tarefas de casa para promover a generalização das aquisições treinadas nas sessões, por exemplo, elogiar pessoas próximas (pai, mãe, colegas de sala etc.). As sessões foram filmadas para fornecer feedback ao desempenho da pesquisadora e permitir eventuais alterações no planejamento da intervenção.

As dez atividades complementares de Treino de Habilidades Sociais em sala - com objetivo de promover a generalização do repertório social envolveram todos os estudantes e os dois professores dos alunos do GHS. No final da aplicação, cada professor recebia instruções verbais para utilizar habilidades que visavam favorecer o desenvolvimento e a aprendizagem de seus alunos (Del Prette \& Del Prette, 2001): a) habilidades de apresentação das atividades escolares, tais como: Explicite o objetivo on produto esperado da atividade; Estabelesa conseqüências para o desempenho; Promova a participação dos alunos por meio de atividades desafiadoras; b) habilidades de transmissão dos conteúdos, tais como: Exponba com clareza; Apresente modelos; Faça perguntas, Faça pausa para o aluno elaborar a resposta; c) habilidades de mediação de interações educativas entre os alunos, tais como: Chame a atenção de um aluno para o desempenho de outro, Solicite e valorize a cooperação, Incentive feedback e o elogio entre eles; Estabeleşa regras de convivencia em sala, inclusive para o uso das palavras por favor, desculpe etc.); d) habilidades de avaliação das atividades propostas, tais como: Explicite os critérios de avaliação; Desenvolva habilidades de auto-avaliação nos alunos. 
Procedimento de promoção do repertório acadêmico

O Programa de Ensino de Leitura e Escrita, fundamentado no método de controle de estímulos, consistiu do primeiro de três módulos que estabelecem uma ordem crescente de complexidade para a promoção da leitura com compreensão e fluência. Nesse primeiro módulo focaliza-se o ensino de palavras de duas e três sílabas simples e regulares do tipo consoante e vogal, com reconhecimento de palavras ensinadas e generalização para palavras novas (recombinação de letras). $O$ segundo e o terceiro módulos, não aplicados na pesquisa, envolvem o ensino de palavras complexas e textos completos, respectivamente. A descrição detalhada do procedimento é encontrada em de Rose et al. (1992) e
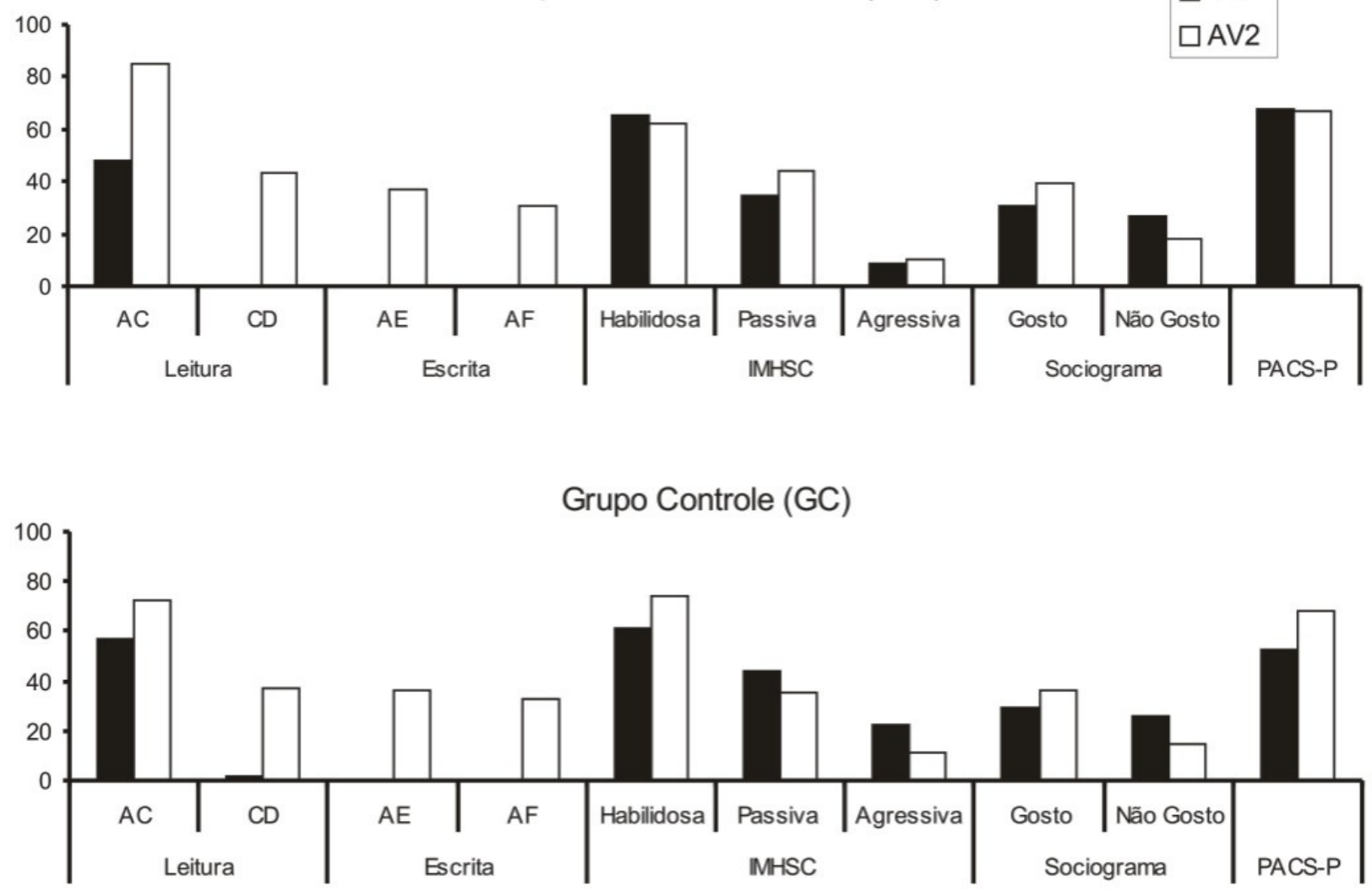

Tipos de Avaliação

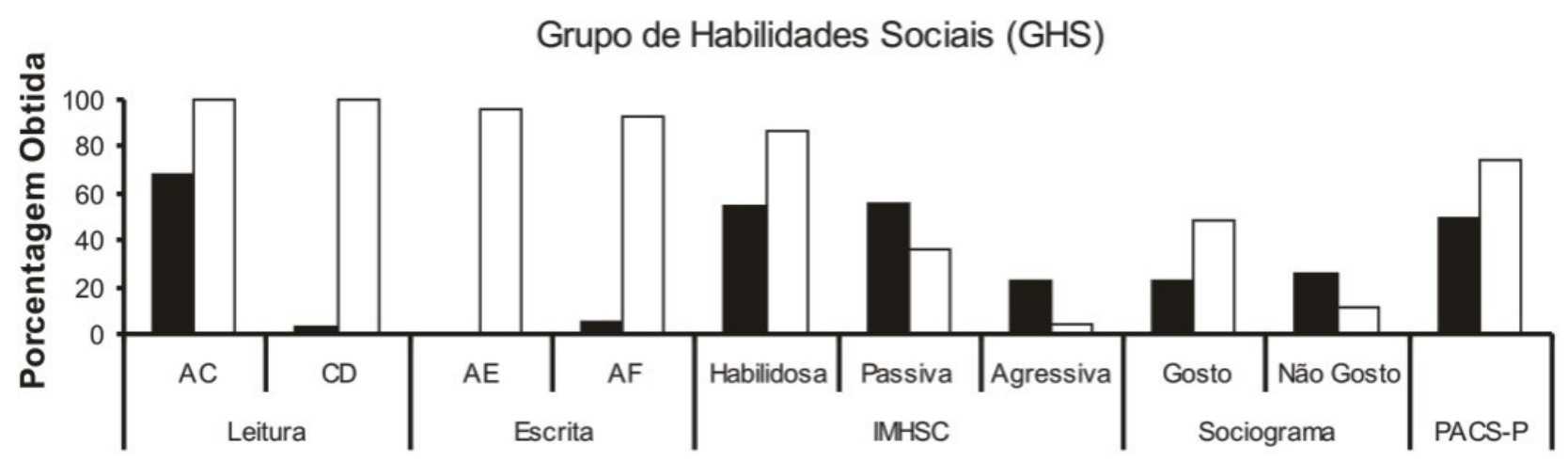

Figura 1 - Percentagem de acertos (indicador acadêmico) e de freqüência relativa (comportamento social) dos três grupos de pesquisa, obtida na Avaliação Inicial (AV1, barras pretas) e Final (AV2, barras vazias) 
em de Rose, de Souza e Hanna (1996).

Para a realização das sessões de intervenção, pequenos grupos de crianças eram transportados por um microônibus até a Unidade de Leitura, onde permaneciam uma hora por dia. Deste tempo, trinta minutos eram utilizados para a condução de sessões de ensino de leitura e escrita, e os outros trinta para a realização de desenhos e jogos em grupo. As sessões de ensino eram realizadas individualmente pelos participantes, os quais eram monitorados por bolsistas do local. Dois participantes deste grupo não concluíram o programa, realizando $70 \%$ dele. A média de intervenção em leitura e escrita realizada pelo GEL, em sete meses de intervenção, foi de 70 sessões (70 horas).

\section{Tratamento dos dados}

Para avaliar o Inventário Multimídia de Habilidades Sociais para Criancas - IMHSC-Del Prette (Del Prette \& Del Prette, 2005b) foram computados os escores de cada criança nas reações habilidosa, passiva e agressiva, que podiam variar de zero a 42 cada, devido aos valores numéricos da escala para cada item (sempre $=2$; às vezes $=1$; nunca $=0)$. Na computação do escore obtido no Protocolo de Avaliação da Competência Social junto ao Professor (Del Prette \& Del Prette, 2003) foram atribuídos os seguintes pontos para cada item (no pólo positivo $=2$; no intermediário $=1$; no negativo $=0$ ), calculando-se o percentual de pontos obtidos pelo aluno em relação à pontuação total possível. Com relação aos dados da Ficha de Avaliação Sociométrica, para cada criança, foi calculada a percentagem das avaliações de "gosto" e "não gosto" recebida pelos colegas da classe. Os dados da Ficha de Avaliação de Leitura e Escrita (Fonseca, 1997) foram computados em termos da porcentagem de acertos das 15 tentativas das tarefas de seleção (AC), nomeação de palavras (CD) e ditado/escrita (AE e AF).

Após a tabulação dos dados individuais, foram calculadas as médias de cada grupo e multiplicadas por 100 para se trabalhar com números inteiros. Com base nesses valores, foi verificada a correlação e/ou a diferença entre os indicadores de repertório acadêmico e social, aplicando-se testes não paramétricos para a comparação entre os três grupos (Kruskal-Wallis) e para a comparação destes dois a dois (Mann-Whitney), como uma tentativa exploratória de verificar a significância dos resultados, mesmo se reconhecendo suas limitações devido ao reduzido número de participantes.

\section{Resultados}

A Figura 1 apresenta os resultados obtidos na Avaliação Inicial (indicada por barras pretas) e Final (indicada por barras vazias) do repertório acadêmico (tarefas de seleção/AC, nomeação de palavras/CD, ditado/AE e escrita/AF) e social (auto-avaliação e avaliação pelos colegas e professores) dos grupos de pesquisa. A Tabela 1 apresenta os resultados estatísticos sobre as diferenças entre as avaliações para os três (Kruskal-Wallis) e para os pares (Mann-Whitney) de grupos.

Com relação ao repertório social do GHS (foco de intervenção), a diferença entre AV2 e AV1 revelou ganhos expressivos no status sociométrico dos participantes (indicações de gosto), na auto-avaliação (respostas habilidosas) e na avaliação do professor. Além disso, foram encontradas oscilações negativas nas respostas passivas e agressivas e nas indicações de não gosto. Os ganhos obtidos pelos participantes desse grupo em leitura e escrita variaram de 32\% (relações AC) a 97\% (relações $\mathrm{CD}$ ). Na maioria dessas análises os resultados favoráveis do GHS foram significativamente superiores aos do GEL e do GC, como se vê na Tabela 1.

Comparando-se visualmente os dados intragrupo (Figura 1), os participantes do GEL apresentaram ganhos em leitura e escrita (foco de intervenção) que variaram de $31 \%$ (relações $\mathrm{AF}$ ) a $43 \%$

Tabela 1 - Comparação das diferenças entre a avaliação final (AV2) e a inicial (AV1) para os grupos (KruskalWallis) e para os grupos dois a dois (Mann-Whitney)

\begin{tabular}{cccccccccc}
\hline \multirow{2}{*}{ Avaliação } & GEL & GHS & GC & \multicolumn{2}{c}{ Kruskal-Wallis } & \multicolumn{2}{c}{ Mann-Withney } & \\
\cline { 2 - 6 } & (AV2-AV1) & (AV2-AV1) & (AV2-AV1) & $\mathrm{H}$ & $\mathrm{p}$ & $\mathrm{W}$ & $\mathrm{p}$ & Conclusão \\
\hline CD & $43 \%$ & $97 \%$ & $35 \%$ & 6,11 & 0,04 & 10 & 0,01 & GHS $>$ GEL \\
& & & & & & & & \\
Habilidosa & $-3 \%$ & $32 \%$ & $13 \%$ & 8,86 & 0,01 & 10 & 0,02 & GHS $>$ GC \\
Passiva & $9 \%$ & $-20 \%$ & $-9 \%$ & 6,22 & 0,04 & 25 & 0,01 & GHS $>$ GC \\
Agressiva & $1 \%$ & $-19 \%$ & $-11 \%$ & - & - & 25 & 0,04 & GEL $>$ GHS \\
\hline
\end{tabular}


(relações CD). Com relação ao repertório social, houve apenas uma pequena diferença nas respostas passivas (11\% mais alta na AV2), no sentido oposto ao esperado, enquanto houve um discreto aumento percentual nas avaliações de "gosto" (8\%) e diminuição nas de "não gosto" (9\%). Na avaliação do professor (PACS-P), os valores foram praticamente iguais nas duas avaliações.

No caso do GC, a diferença entre AV2 e AV1 revelou ganhos no percentual de respostas habilidosas, nas indicações de "gosto" e na avaliação do professor. Por outro lado, no percentual de respostas passivas e agressivas e nas avaliações de "não gosto" verificaramse valores mais reduzidos na AV2. Os ganhos obtidos nas relações de leitura e escrita variaram de 15\% (em AC) e $36 \%$ (em AE).

\section{Discussão}

Comparando-se a Avaliação Inicial (AV1) e Final (AV2) dos três grupos, os resultados indicam aquisições do grupo experimental GHS que sugerem uma relação de funcionalidade entre o desenvolvimento interpessoal e acadêmico, embora não seja conclusivo quanto aos efeitos da aquisição acadêmica sobre o repertório social. Além dos ganhos mais significativos em leitura e escrita, o grupo apresentou melhorias mais acentuadas que o GEL e GC no repertório social, em termos da auto-avaliação e avaliação pelos professores. Os dados permitem supor que a auto-avaliação e a avaliação por outros (colegas e professores) são processos interligados no que se refere ao desempenho social e que a competência acadêmica pode estar associada à melhoria de ambas. Para autores anteriormente citados (Vaughn \& Hogan, 1990; Vaughn, McIntosh, \& Spencer-Rowe, 1991), as interações sociais que o estudante mantém com outros significativos repercutem em sua auto-avaliação social afetando também o desempenho acadêmico. Portanto, a melhoria na avaliação dos colegas e professores pode ter sido um reflexo de relações interpessoais mais satisfatórias dos participantes, o que, por sua vez, pode ter contribuído para sua compreensão social dos comportamentos socialmente desejáveis, o que, segundo Del Prette e Del Prette (2005b), constitui um componente importante da competência social.

Alguns procedimentos, adotados pela pesquisadora, podem ter facilitado a obtenção de resultados positivos com o GHS, tais como: a realização de atividades complementares de treino de habilidades sociais em sala de aula, envolvendo todos os alunos, com o objetivo de promover a generalização do repertório social e reforçar o comportamento aprendido nas sessões (Del Prette \& Del Prette, 1999); a conclusão de todos os objetivos de intervenção com cada um dos participantes do grupo; e a utilização de pares sem distúrbios de aprendizagem na intervenção, os quais podem ter atuado como agentes efetivos de mudança funcionando como bons modelos a seus pares com problemas (Vaughn, 1991).

Ainda que o GEL não tenha produzido efeitos sobre o repertório social das crianças, não se pode descartar, de imediato, essa possibilidade. Apesar da intervenção nos dois grupos (GEL e GHS) ter envolvido praticamente a mesma duração em termos de horas/sessão, a quantidade de aquisição acadêmica do GEL (já que foi implementado somente o primeiro módulo do programa e, ainda, porque esse módulo inicial foi completado por somente metade dos participantes) pode não ter sido suficiente para produzir os efeitos esperados no repertório social desses estudantes. Ao finalizar o Módulo 1, que tem como objetivo o ensino de palavras de duas e três sílabas simples tipo consoante e vogal, de Rose et al. (1992) consideram que o aluno apenas iniciou a aquisição de leitura e escrita e está apto para realizar atividades mais complexas envolvidas no repertório do leitor proficiente. De fato, ler e escrever envolvem a capacidade de compreender e expressar idéias bem como de comunicar-se mediante o uso da linguagem escrita. Assim, somente quando consegue realizar a leitura e a produção de textos com compreensão, é que se pode afirmar, conforme De Rose, de Souza e Hanna (1996), que o estudante adquiriu estes repertórios em seu comportamento. Portanto, para verificar os efeitos da aquisição de repertório acadêmico sobre o repertório social de estudantes com dificuldades de aprendizagem, pode ser importante, em estudos futuros, a conclusão dos três módulos do Programa de Ensino (ensino de palavras simples, complexas e textos completos, respectivamente).

Algumas aquisições verificadas no repertório do GC podem ser explicadas por outros fatores inerentes ao contexto de intervenção e à dificuldade de completo controle em situação natural. Como exemplo, o fornecimento de instruções verbais aos professores pode ter contribuído, ainda que em menor monta, para os ganhos verificados no repertório social e acadêmico dos alunos do GC. Além disso, no caso específico deste estudo, os professores participantes reuniam um conjunto de habilidades sociais profissionais que, conforme Del Prette e Del Prette (1997a) são importantes para a promoção da aprendizagem e desenvolvimento interpessoal dos alunos: percepção das demandas da sala de aula, flexibilidade para mudar a ação em função dessas demandas, acuracidade de observação, 
análise e discriminação do progresso dos alunos em termos de suas capacidades reais e potenciais, além de outras como expressar opiniões, dar e receber feedback etc.

Uma outra condição bastante restritiva para estudos experimentais em contexto natural refere-se à necessidade de sua delimitação ao longo de um ano letivo, já que muitas outras variáveis entram em cena quando se passa para um segundo ano (como novos colegas e professores). Assim, é importante um cronograma altamente delimitado, com avaliações iniciais nos primeiros meses do ano letivo e, em seguida, promover o treinamento para, então, terminá-lo no final do mesmo ano.

Uma limitação evidente deste estudo refere-se à quantidade de sujeitos participantes, o que também é bastante difícil de ser superado em pesquisas desse tipo. Apesar de se iniciar com maior número de alunos, fatores não controláveis pelo pesquisador (como o abandono da escola e a mudança de domicílio de algumas crianças) também ocorreram. Em estudos futuros, sob condições mais favoráveis, a inclusão de um número maior de participantes poderia trazer dados adicionais relevantes para as questões aqui tratadas.

A realização das pesquisas sugeridas neste trabalho exigirá do pesquisador lidar com diversos empecilhos em trabalhos de intervenção como este. Os mais comuns se referem à falta de assiduidade dos alunos na escola e à dificuldade de obtenção da assinatura do termo de consentimento pelos pais ou responsáveis. Particularmente neste trabalho, esses dois aspectos também dificultaram a reunião de um número maior de participantes, a realização das avaliações iniciais nos primeiros meses do ano letivo e a conclusão do programa de leitura e escrita com os participantes. Neste sentido, torna-se emergencial uma maior integração famíliaescola, como sugere Feitosa (2003), em que os pais possam participar ativamente das decisões relacionadas à educação de seus filhos, conscientizando-se acerca de suas dificuldades acadêmicas e da necessidade de recursos específicos para o ideal desenvolvimento das potencialidades destes alunos.

A existência da relação funcional entre a promoção de habilidades sociais e ganhos no desempenho acadêmico remete à necessidade de se prover condições escolares para o desenvolvimento socioemocional dos alunos como uma estratégia importante para a superação das dificuldades de aprendizagem. Pais, professores e profissionais comprometidos com esta tarefa garantirão uma educação mais efetiva, pautada no respeito aos direitos da criança e na busca da qualidade de vida comunitária.

\section{Referências}

Almeida, C. S. (1984). Análise dos motivos de encaminhamento de alunos de classes comuns a classes especiais de escolas públicas de primeiro grau (Dissertação de Mestrado). São Carlos: Programa de Pós-Graduação em Educação Especial, Universidade Federal de São Carlos.

Almeida, S. F. C., Rabelo, L. M., Cabral, V. S., Moura, E. R. O., Barreto, M. S. F. \& Barbosa, H. (1995). Concepções e práticas de psicólogos escolares acerca das dificuldades de aprendizagem. Psicologia: Teoria e Pesquisa, 11, 117-134.

Brasil (1994). Política Nacional de Educação Especial. Livro1/MEC/SSESP. Brasília: Secretaria.

Brasil, Secretaria de Educação Especial (1994). Encaminhamentos de Alunos do Ensino Regular para o Atendimento Especializado. MEC/Ministério da Educação e do Desporto - Série Diretrizes - Brasília: MEC/SEESP.

Coie, J. D., Dodge, K. A. \& Coppotelli, H. (1982). Dimensions and types of social status: A cross-age perspective. Developmental Psychology, 18, 557-570.

Coll, C., \& Sole, I. (1995). A interação professor/aluno no processo de ensino aprendizagem. In: C. Coll, J. Palácios \& A. Marchesi, (Orgs.). Desenvolvimento psicológico e educação: Necessidades educativas especiais e aprendizagem escolar. Porto Alegre: Artes Médicas.

De Rose, J. C., De Souza, D. G. \& Hanna, E. S. (1996). Teaching reading and spelling: Exclusion and stimulus equivalence. Journal of Applied Behavior Analysis, 29, 451469.

De Rose, J. C., De Souza, D. G., Rossito, A. L., \& de Rose, T. M. S. (1992). Stimulus equivalence and generalization in reading after matching to sample by exclusion. Em S. C. Hayes \& L. J. Hayes (Orgs.). Understanding verbal relations (pp. 69-82). Reno, EUA: Context Press.

Del Prette, A. \& Del Prette, Z. A. P. (1997a). Habilidades sociais e construção de conhecimento em contexto escolar. Em D. R. Zamignani (Org.). Sobre comportamento e cognição: a aplicação da análise do comportamento e da terapia cognitivo-comportamental no hospital geral e nos transtornos psiquiatricos (pp.234-250). Santo André: ARBytes.

Del Prette, A. \& Del Prette, Z. A. P. (2001). Psicologia das relações interpessoais: vivências para o trabalho em grupo. Petrópolis: Vozes.

Del Prette, Z. A. P. \& Del Prette, A. (1999). Psicologia das habilidades sociais: terapia e educação. Petrópolis: Vozes. 
Del Prette, Z. A. P. \& Del Prette, A. (2003). Habilidades sociais e dificuldades de aprendizagem: Teoria e pesquisa sob um enfoque multimodal. Em A. Del Prette \& A. P. Del Prette (Orgs.). Habilidades sociais, desenvolvimento e aprendizagem: questões conceituais, avaliação e intervenção (pp. 167-206). Campinas: Alínea.

Del Prette, Z. A. P. \& Del Prette, A. (2005a). Treinamento de habilidades sociais na infância: teoria e prática. Petrópolis: Vozes.

Del Prette, Z. A. P. \& Del Prette. A. (2005b). Sistema Multimidia de Habilidades Sociais para Crianças (IMHSCDel-Prette): manual. Petrópolis: Vozes.

Del Prette, Z. A. P., Del Prette, A. \& Gresham, F. M (s. d.). Social skills and learning problems: A multimethod comparison with a Brazilian Sample. Texto disponível com os autores.

Elliott C., Pring, T. \& Bunning K. (2002). Social skills training for adolescents with intellectual disabilities: A cautionary note. Journal of Applied Research in Intellectual Disabilities, 15, 91-96.

Fad, K. S. (1989). The fast track to success: Social behavioral skills. Intervention in School and Clinic, 3, 3942.

Feitosa, F. B. (2003). Relação familia-escola: como pais e professores avaliam e reagem ao repertório social de crianças com $e$ sem dificuldades de aprendizagem (Dissertação de Mestrado). São Carlos: Programa de Pós-Graduação em Educação Especial, Universidade Federal de São Carlos (SP).

Fonseca, M. L. (1997). Diagnóstico de repertórios iniciais de leitura e escrita: uma análise baseada na noção de redes de relações de equivalência (Dissertação de Mestrado). São Carlos: Programa de Pós-Graduação em Educação Especial da UFSCar.

Garotti, M., de Souza, D., De Rose, J., Molina, R. C. \& Gil, M. S. (2000). Reorganization of equivalence classes after reversal of baseline relations. The Psichological Record, 50, 35-48.

Gresham, F. M. (1992). Social skills and learning disabilities: Causal, concomitant or correlational? School Psychological Review, 21, 348-360.

Gresham, F. M. (1995). Best practices in social skills training. Em A. Thomas e J. Grimes (Orgs.). Best Practices in School Psychology-III (pp. 1021-1030). Washington DC: The National Association of School Psychologists.

Gresham, F. M. (2002). Social skills assessment and instruction for students with emotional and behavioral disorders. Em K. L. Lane, F. M. Gresham e T. E.
O'Shaughnessy (Orgs.). Children with or at risk for emotional and behavioral disorders (pp. 177-194). Boston: Allyn \& Bacon.

Haynes, S. \& O’Brien, W. (1990). Functional analysis in behavior therapy. Clinical Psychology Review, 10, 649-668.

Hildebrand, F. C. (2000). Dificuldades de aprendizagem: habilidades sociais presentes nas interações (Dissertação de Mestrado). São Carlos: Programa de Pós-Graduação em Educação Especial da UFSCar.

Magalhães, T., Del Prette, A. \& Del Prette, Z. A. P. (2005). Inventário Multimídia de Habilidades Sociais para Crianças (IMHSC-Del-Prette): dados psicométricos adicionais [Resumos]. II Congresso Brasileiro de Educação Especial e II Encontro da Associação dos Pesquisadores em Educaşão Especial: Construindo o Conhecimento, São Carlos (SP), pp. 362-363.

Malecki, C. K. \& Elliott, S. N. (2002). Children's social behaviors as predictors of academic achievement: A longitudinal analysis. School Psychology Quarterly, 17, 1-23.

Maluf, M. R. \& Bardelli, C. (1991). As causas do fracasso escolar na perspectiva de professoras e alunos de uma escola de primeiro grau. Psicologia: Teoria e Pesquisa, 7 , 255-262.

Marturano, E. M. \& Loureiro, S. M. (2003). O desenvolvimento socioemocional $\mathrm{e}$ as queixas escolares. Em Del Prette, A. \& Del Prette, Z. A. P. (Orgs.). Habilidades sociais, desenvolvimento e aprendizagem: questôes conceituais, avaliação e intervenção (pp. 259-291). Campinas: Alínea

Marturano, E. M., Linhares, M. B. M. \& Parreira, V. L. C. (1993). Problemas emocionais associados a dificuldades na aprendizagem escolar. Medicina Ribeirão Preto, 26, 161-175.

McClelland. M. M., Morrison, F. J. \& Holmes, D. L. (2000). Children at risk for early academic problems: The role of learning-related social skills. Early Childhood Research Quarterly, 15, 307-329.

Mello, M. H. S. (2004). Crianças com dificuldades de interação no ambiente escolar: Uma intervenção multifocal (Dissertação de Mestrado). São Paulo: Programa de Pós-Graduação em Psicologia Clínica, Universidade de São Paulo.

Molina, R. C. M. (2003). Habilidades sociais e dificuldades de aprendizagem: Uma análise funcional. (Dissertação de Mestrado). São Carlos: Programa de Pós-Graduação em Educação Especial da UFSCar.

Nunes, L. R. O., Ferreira, J. R., Glat, R. \& Mendes, E. G. (1998). Questôes atuais em educação especial: a pesquisa em educação especial na pós-graduação. vol. 3. Rio de Janeiro: Sette Letras. 
O'Shaughnessy, T. E., Lane, K. L., Gresham, F. M. \& Beebe-Frankenberger, M. E. (2002). Students with at risk for learning and emotional-behavioral difficulties: An integrated system of prevention and intervention. Em K. L. Lane, F. M. Gresham \& T. E. O'Shaughnessy (Orgs.). Children with or at risk for emotional and behavioral disorders (pp. 3-17). Boston: Allyn \& Bacon.

Pasquali, L. (1996). (Org.). Teoria e métodos de medida em ciências do comportamento. Brasilia: Laboratório de Pesquisa em Avaliação e Medida, Instituto de Psicologia/UnB: INEP.

Pinheiro, M. I. S., Haase, V. G., Del Prette, A., Amarante, C. L. D. \& Del Prette, Z. A. P. (no prelo). Treinamento de habilidades sociais educativas para pais de crianças com problemas de comportamento. Psicologia: Reflexão e Crítica.

Pontecorvo, C. (s. d.). Social context, semiotic mediation and forms of discourse in constructing knowledge at school. (Mimeo).

Romero, A. (1995). As relações sociais das crianças com dificuldades de aprendizagem. Em C. Coll, J. Palácios \& A. Marchesi (Orgs.). Desenvolvimento psicológico e educação: Necessidades educativas especiais e aprendiragem escolar (pp. 71-82). Porto Alegre: Artes Médicas.

Sánchez, J. N. G. (2004). Dificuldades de aprendizagem e intervenção psicopedagógica. Porto Alegre: Artmed.
Souza, M. P. R. de \& Machado, A. M. M. (1997). As crianças excluídas da escola: um alerta para a Psicologia. Em A. M. M. Machado \& M. P. R. de Souza (Orgs.). Psicologia escolar: novos rumos. São Paulo: Casa do Psicólogo - Psicologia da Educação.

Stone, W. L. \& La Greca, A. M. (1990). The social status of children with LD: A reexamination. Journal of Learning Disabilities, 23, 32-37.

Swanson, H. L. \& Malone, S. (1992). Social skills and learning disabilities: A meta-analysis of the literature. School Psychology Review, 21, 427-443.

Vaughn, S. (1991). Social skills enhancement in students with learning disabilities. Learning about Learning disabilities, 10, 55-69.

Vaughn, S. \& Hogan, A. (1990). Social competence and learning disabilities: A prospective study. Em H. L. Swanson e B. K. Keogh (Orgs.). Learning Disabilities: Theoretical and Research Issues (pp. 175-191). New Jersey: Lawrence Erlbaum.

Vaughn, S., McIntosh, R. \& Spencer-Rowe, D. (1991). Peer rejection is a stubborn thing. Learning Disabilities Research \& Practice, 6, 83-88.

Recebido em outubro de 2005 Reformulado em janeiro de 2006 Aprovado em fevereiro de 2006

Sobre as autoras:

Renata Cristina Moreno Molina é psicóloga, mestre em Educação Especial e doutoranda em Educação Especial pelo Programa de Pós-Graduação em Educação Especial da UFSCar. A Psicologia das Habilidades Sociais constituem alvo de pesquisa da autora atualmente.

Zilda Aparecida Pereira Del Prette é psicóloga, mestre em Psicologia Comunitária, doutora em Psicologia Experimental e pós-doutora em Habilidades Sociais. Atualmente é professora titular do Departamento de Psicologia da UFSCar, pesquisadora do CNPq, professora e orientadora do Programa de Pós-Graduação em Educação Especial (UFSCar) e em Psicologia (USP/Ribeirão Preto). Ministra disciplinas sobre Relações Interpessoais e Habilidades Sociais na graduação e pós-graduação, orientando pesquisas e estágios na área. 